\title{
根际低氧逆境对网纹甜瓜幼苗生长及根系 呼吸代谢途径的影响
}

\author{
孙艳军 郭世荣* 胡晓辉 高洪波
}

（南京农业大学园艺学院, 南京 210095)

\begin{abstract}
摘 要 以耐低氧性具有明显差异的两个网纹甜瓜 (Cucumis melo var. raticulalus) 品种为试材, 研究了根际低氧胁迫 下幼苗生长、根系活力及根系呼吸关键酶活性的变化。结果表明, 根际低氧胁迫下, 两品种幼苗生长均受到明显抑 制, 而根系活力升高; 根系 PDC 活性两品种均显著提高, 品种间无显著差异; MDH 活性两品种均显著降低, 且耐低 氧性弱的“西域一号”下降幅度较大; 根系 ADH 和 LDH 活性两品种均显著提高, 耐低氧性强的“东方星光”ADH 活性 增加的幅度显著高于而低氧性弱的“西域一号”, 而“西域一号” LDH 活性增加幅度显著高于 ‘东方星光”。说明 “东 方星光”在低氧胁迫下能保持较高的有氧呼吸水平, 无氧呼吸的主要途径为乙醇发酵, 而 “西域一号” 在低氧胁迫下 无氧呼吸的主要途径为乳酸发酵。
\end{abstract}

关键词低氧胁迫网纹甜瓜无氧呼吸

\section{EFFECT OF ROOT-ZONE HYPOXIA STRESS ON GROWTH AND RESPIRATORY METABOLISM PATHWAY OF MUSKMELON SEEDLING ROOTS}

\author{
SUN Yan-Jun GUO Shi-Rong ${ }^{*}$ HU Xiao-Hui and GAO Hong-Bo \\ (Department of Horticulture, Nanjing Agricultural University, Nanjing 210095, China)
}

\begin{abstract}
In this paper, two muskmelon cultivars, 'Xiyu 1' with low hypoxia-resistance and 'Dongfangxingguang' with high hypoxia-resistance, were used to study the changes in the activities in PDC, ADH, LDH and MDH under hypoxia stress. ( DO at $2 \pm 0.20 \mathrm{mg} \bullet \mathrm{L}^{-1}$ ). When seedlings grew to 3 rd euphylla, they were cultured in a Hoagland nutrient solution and divided into two sets. One set of seedlings was exposed to normal dissolved oxygen levels (DO) using vigorous aeration to maintain DO levels at $8.0-8.5 \mathrm{mg}^{\bullet} \mathrm{L}^{-1}$, and the other set was treated with hypoxia stress using a DO analyzer (QUANTUM-25) to keep levels at $2.0 \pm 0.2 \mathrm{mg} \mathrm{L}^{-1}$. The results showed that seedling growth of both cultivars were significantly inhibited and the root activity increased under hypoxic conditions. The PDC activity in roots of both seedlings increased significantly under hypoxia stress, but there were no obvious differences between the two cultivars. The MDH activity in roots of both seedlings decreased significantly under hypoxia stress and the decrease of activity of 'Xiyu 1' was much more than that of 'Dengfangxingguang'. The activities of ADH and LDH of both seedlings increased significantly under hypoxia stress. The increase in the activity of ADH in 'Dongfangxingguang' was more than that of 'Xiyu 1', while the increase of the activity of LDH in 'Xiyu 1' was more than that of 'Dongfangxingguang' . The results suggested that 'Dongfangxingguang' could maintain high levels of aerobic respiration, and ethanol fermentation was the primary pathway of anaerobic respiration under hypoxic stress whereas lactate fermentation was the leading pathway of anaerobic respiration under hypoxia stress in 'Xiyu 1'.
\end{abstract}

Key words Hypoxia stress, Muskmelon (Cucumis melo var. raticulalus), Anaerobic respiration

$\mathrm{O}_{2}$ 作为呼吸作用电子传递链的末端电子受体, 驱动 $\mathrm{ATP}$ 和 $\mathrm{NAD}(\mathrm{P})+$ 的合成, 维持细胞生长所需要 的还原力, 构成整个植物体生命代谢的核心, 是植物 正常生长发育必不可少的条件。洪涝灾害、灌水过 多或无土栽培时常常造成作物根际氧气缺乏, 低氧
逆境已成为作物生产主要限制因子之一, 因此, 研究 低氧逆境胁迫对作物生长和生理代谢的变化具有重 要的意义。研究表明, 低氧条件下, 玉米( Zea mays) (Johnson et al.，1994)、小麦 ( Triticum aestivum) (Biemelt et al.,1998) 等有氧呼吸受抑制, 诱导无氧 
呼吸代谢基因的表达, 以无氧呼吸产生的能量来暂 时维持细胞的生命活动。但植物对低氧逆境的反应 因物种而异, 即使同一物种不同基因型品种间也存 在显著差异。

网纹甜瓜 (Cucumis melo var. raticulalus) 已列入 世界十大主要水果,也成为我国无土栽培的主栽作 物之一, 但无土栽培的网纹甜瓜存在根系耗氧快、对 根际低氧逆境敏感等问题, 严重制约网纹甜瓜无土 栽培的大面积生产 (郭世荣, 2003)。为了逐步揭示 网纹甜瓜植株低氧伤害和耐性机理, 本文以耐低氧 性具有明显差异的两个网纹甜瓜品种为试材, 在准 确控制营养液溶氧浓度 (Dissolved oxygen, DO) 条件 下, 研究低氧逆境对幼苗生长及根系呼吸代谢关键 酶活性影响,初步阐明低氧逆境下网纹甜瓜植株的 呼吸代谢途径的变化规律。

\section{1 材料和方法}

\section{1 植株培养和处理}

试验于 2004 年 $4 \sim 6$ 月和 9 10 月在南京农业 大学玻璃温室内进行。以低氧耐性较强的网纹甜瓜 品种 “东方星光” ( Dongfangxingguang', 东方正大公 司提供, 引自泰国)和耐性较弱的品种“西域一号, ('Xiyu 1', 新疆西域种子公司提供) 为材料。种子 消毒后催芽, 发芽的种子播于装有石英砂的 $8 \mathrm{~cm} \times 8$ $\mathrm{cm}$ 营养针中育苗, 温室昼温 $27 \sim 30{ }^{\circ} \mathrm{C}$, 夜温 $16 \sim 18$ ${ }^{\circ} \mathrm{C}$, 待第一片真叶完全展开后开始浇灌 $1 / 2$ 倍 Hoagland 营养液 $(\mathrm{pH}$ 值 $6.3 \pm 0.1$, EC 值 $1.2 \sim 1.4$ $\left.\mathrm{mS} \cdot \mathrm{cm}^{-1}\right)$ 。待幼苗达三叶一心时, 选整齐一致的幼 苗(每品种 30 株) 定植于装有 100 L 1 倍 Hoagland 营 养液 ( $\mathrm{pH}$ 值 $6.3 \pm 0.1$, EC 值 $1.9 \sim 2.0 \mathrm{mS} \mathrm{cm}^{-1}$ ) 的 栽培槽中, 气䈋正常通气( DO 维持在 $8.0 \sim 8.5 \mathrm{mg}$ • $\mathrm{L}^{-1}$ ), 预培养 $2 \mathrm{~d}$ 后, 将“东方星光”和 “西域一号”植 株各分成两部分进行处理: 以正常通气营养液栽培 为对照 (CK), 低氧处理区, 用溶氧浓度调节器(美国 Quantum, Q25D 型) 调节营养液的溶氧浓度为 2.0 $\mathrm{mg} \bullet \mathrm{L}^{-1}\left(\right.$ 误差小于 $\left.\pm 0.20 \mathrm{mg} \cdot \mathrm{L}^{-1}\right)$ 。每处理重复 3 次。

\section{2 测定项目及方法}

1.2.1 生长指标的测定

在处理 $8 \mathrm{~d}$ 后取植株测定株高和根长, 擦干水 分后将植株分为地下部和地上部分别测定其鲜重, 然后在 $105{ }^{\circ} \mathrm{C}$ 下杀青 $15 \mathrm{~min}, 75{ }^{\circ} \mathrm{C}$ 下烘干至恒重, 测 定其干重,并计算含水率。
参照李合生(2000)的 TTC 法, 以四氮唑的还原 强度表示根系活力的大小。

\subsubsection{PDC、ADH、LDH 活性的测定}

\subsubsection{1 酶液的提取}

剪取中部根系, 称取 $0.4 \mathrm{~g}$ 左右于研钵中, 加入 预冷的 $1.6 \mathrm{ml}$ 的 Tris-HCl (pH6.8) 提取液 (含 5 $\mathrm{mmol} \cdot \mathrm{L}^{-1} \mathrm{MgCl}_{2}, 5 \mathrm{mmol} \cdot \mathrm{L}^{-1} \beta$-颈基乙醇, $15 \%$ 甘油, 1 $\mathrm{mmol} \cdot \mathrm{L}^{-1} \mathrm{EDTA}, 1 \mathrm{mmol} \cdot \mathrm{L}^{-1} \mathrm{EGTA}$ 和 $0.1 \mathrm{mmol} \cdot \mathrm{L}^{-1}$ 苯甲基磺酰氟), 于冰浴研磨后, $4{ }^{\circ} \mathrm{C} 12000 \times g$ 离 心 $20 \mathrm{~min}$, 上清液为粗酶液 (Mustroph \& Albrecht, 2003)。

\subsubsection{2 酶活性的测定}

丙酮酸脱羧酶 (PDC) 活性: 参考 Waters 等 (1991)的方法, 并略有改进。反应混合液含 $940 \mu \mathrm{l}$ $50 \mathrm{mmol} \cdot \mathrm{L}^{-1} \operatorname{MES}(2-[\mathrm{N}$-morpholine $]$-ethane-sulphonic acid)缓冲液 ( $\mathrm{pH} 6.8$, 含 $25 \mathrm{mmol} \cdot \mathrm{L}^{-1} \mathrm{NaCl}, 1 \mathrm{mmol} \cdot$ $\mathrm{L}^{-1} \mathrm{MgCl}_{2}, 0.5 \mathrm{mmol} \cdot \mathrm{L}^{-1} \mathrm{TPP}, 2 \mathrm{mmol} \cdot \mathrm{L}^{-1} \mathrm{DTT}, 0.17$ $\mathrm{mmol} \cdot \mathrm{L}^{-1} \mathrm{NADH}, 50 \mathrm{mmol} \cdot \mathrm{L}^{-1}$ 草氨酸钠, $10 \mathrm{U}$ $\mathrm{ADH}), 50 \mu \mathrm{l}$ 酶提取液, 用 $10 \mu \mathrm{l} 10 \mathrm{mmol} \cdot \mathrm{L}^{-1}$ 丙酮酸 启动反应, 测定 $340 \mathrm{~nm}$ 波长吸光值的变化。

乙醇脱氢酶 (ADH): 参考 Waters 等 (1991) 的方 法, 并略有改进。反应混合液含 $940 \mu \mathrm{l} 50 \mathrm{mmol} \cdot \mathrm{L}^{-1}$ TES( N-tris (hydroxymethyl)methyl-2-aminoethanesul fonic acid）缓冲液 $\left(\mathrm{pH} 7.5\right.$, 含 $0.17 \mathrm{mmol} \cdot \mathrm{L}^{-1} \mathrm{NADH}$ ), 50 $\mu \mathrm{l}$ 酶提取液, 用 $10 \mu \mathrm{l}$ 乙醛 $(40 \%)$ 启动反应, 测定 340 $\mathrm{nm}$ 波长吸光值的变化。

乳酸脱氢酶( LDH) 活性: 按 Bergmeyer(1983) 的 方法测定。反应混合液含 $2.5 \mathrm{ml}$ Tris-NaCl-NADH 缓 冲液 ( $\mathrm{pH} 7.2$, 含 $0.22 \mathrm{mmol} \cdot \mathrm{L}^{-1} \mathrm{NADH}$ ), $150 \mu \mathrm{l}$ 酶提 取液, 用 $0.5 \mathrm{ml}$ Tris-NaCl-丙酮酸 $\left(\right.$ 含 $0.01 \mathrm{~mol} \cdot \mathrm{L}^{-1}$ 丙 酮酸的 $80 \mathrm{mmol} \cdot \mathrm{L}^{-1} \mathrm{Tris}-\mathrm{NaCl}$ 缓冲液) 启动反应, 测 定 $340 \mathrm{~nm}$ 波长吸光值的变化。

\subsubsection{MDH 活性的测定}

酶液的提取: 按欧阳光察(薛应龙, 1985)的方法 并加以适当调整, 称取 $0.4 \mathrm{~g}$ 左右甜瓜根系于研钵 中, 加入预冷的 $1.6 \mathrm{ml}$ 的 Tris-HCl ( $\mathrm{pH} 8.3$ ) 提取液 (含 $5 \mathrm{mmol} \cdot \mathrm{L}^{-1} \mathrm{MgCl}_{2}, 25 \mathrm{mmol} \cdot \mathrm{L}^{-1} \beta$-颈基乙醇, 5 $\left.\mathrm{mmol} \cdot \mathrm{L}^{-1} \mathrm{EDTA}\right)$, 于冰浴研磨, $4{ }^{\circ} \mathrm{C} 12000 \times \mathrm{g}$ 离心 $20 \mathrm{~min}$, 上清液为粗酶液。

MDH 活性的测定: 按欧阳光察 (薛应龙, 1985) 的方法并加以适当调整。反应混合液含 $880 \mu \mathrm{l} 25$ $\mathrm{mmol} \cdot \mathrm{L}^{-1}$ Tris-HCl 缓冲液 $\left(\mathrm{pH} 8.0\right.$, 含 $1 \mathrm{mmol} \cdot \mathrm{L}^{-1}$ EDTA, $\left.0.2 \mathrm{mmol} \cdot \mathrm{L}^{-1} \mathrm{NADH}\right), 20 \mu \mathrm{l}$ 酶提取液, 用 100 $\mu \mathrm{l} 0.5 \mathrm{mmol} \cdot \mathrm{L}^{-1}$ 草酰乙酸启动反应, 测定 $340 \mathrm{~nm}$ 波 
长吸光值的变化。

酶活力以每毫克酶蛋白每分钟 NADH 变化的微 摩尔数来表示, 蛋白含量参照 $\operatorname{Bradford}(1976$ ) 的考马 斯亮兰 G250 法测定。

测定结果采用 SAS 软件 Duncan 多重比较法进 行统计分析。

\section{2 结果与分析}

\section{1 低氧胁迫对幼苗生长的影响}

由表 1 可以看出, 低氧胁迫处理 $8 \mathrm{~d}$ 后, 与通气 对照相比, ‘东方星光”和 ‘西域一号’两个品种幼苗 地下部的生长受到严重抑制, 表现为根长降低、鲜
重、干重显著下降, 两个品种根长较通气对照分别下 降 $36.50 \%$ 和 $37.93 \%$, 鲜重分别下降 $19.28 \%$ 和 $27.61 \%$, 干重分别下降 $10.19 \%$ 和 $12.54 \%$, 含水率 稍有降低。品种间 “西域一号” 受低氧胁迫的抑制更 明显。

由表 2 可以看出, 低氧胁迫处理 $8 \mathrm{~d}$ 后, 与通气 对照相比, “东方星光” 和 “西域一号”两个品种幼苗 地上部的生长同样受到严重抑制, 表现为株高降低, 鲜重、干重显著下降,和通气对照相比,两品种株高分 别降低 $19.80 \%$ 和 $21.71 \%$, 鲜重分别下降 $26.06 \%$ 和 $36.30 \%$, 干重分别下降 $21.85 \%$ 和 $27.00 \%$, 含水率下 降。品种间 ‘西域一号”受低氧胁迫的抑制更明显。

表 1 低氧胁迫对网纹甜瓜地下部生长的影响

Table 1 Effect of hypoxia stress on subterraneous growth of muskmelon seedlings

\begin{tabular}{|c|c|c|c|c|c|}
\hline $\begin{array}{c}\text { 品种 } \\
\text { Cultivars }\end{array}$ & $\begin{array}{c}\text { 处理 } \\
\text { Treatment }\end{array}$ & $\begin{array}{c}\text { 根长 } \\
\text { Root length } \\
(\mathrm{cm})\end{array}$ & $\begin{array}{c}\text { 地下部鲜重 } \\
\text { Subterraneous } \\
\text { fresh weight }\left(\mathrm{g} \bullet \text { plant }^{-1}\right)\end{array}$ & $\begin{array}{c}\text { 地下部干重 } \\
\text { Subterraneous } \\
\text { dry weight }\left(\mathrm{g} \bullet \text { plant }^{-1}\right)\end{array}$ & $\begin{array}{c}\text { 地下部含水率 } \\
\text { Subterraneous } \\
\text { water content }(\%)\end{array}$ \\
\hline ‘东方星光' & 通气对照 CK & $46.44 \pm 0.91$ & $6.41 \pm 0.15$ & $0.21 \pm 0.01$ & $96.74 \pm 0.03$ \\
\hline 'Dongfangxingguang' & 低氧胁迫 Hypoxia stress & $29.49 \pm 0.72$ & $5.17 \pm 0.05$ & $0.19 \pm 0.01$ & $96.36 \pm 0.03$ \\
\hline ‘西域一号” & 通气对照 CK & $55.57 \pm 0.84$ & $7.47 \pm 0.10$ & $0.24 \pm 0.01$ & $96.79 \pm 0.05$ \\
\hline 'Xiyu 1' & 低氧胁迫 Hypoxia stress & $34.50 \pm 0.75$ & $5.41 \pm 0.08$ & $0.21 \pm 0.01$ & $96.12 \pm 0.08$ \\
\hline
\end{tabular}

表 2 低氧胁迫对网纹甜瓜幼苗地上部生长的影响

Table 2 Effect of hypoxia stress on supraterraneous growth of muskmelon seedlings

\begin{tabular}{|c|c|c|c|c|c|}
\hline $\begin{array}{c}\text { 品种 } \\
\text { Cultivars }\end{array}$ & $\begin{array}{c}\text { 处理 } \\
\text { Treatment }\end{array}$ & $\begin{array}{c}\text { 株高 } \\
\text { Plant height } \\
(\mathrm{cm})\end{array}$ & $\begin{array}{c}\text { 地下部鲜重 } \\
\text { Subterraneous } \\
\text { fresh weight }\left(\mathrm{g} \bullet \text { plant }^{-1}\right)\end{array}$ & $\begin{array}{c}\text { 地下部干重 } \\
\text { Subterraneous } \\
\text { dry weight }\left(g \bullet \text { plant }^{-1}\right)\end{array}$ & $\begin{array}{c}\text { 地下部含水率 } \\
\text { Subterraneous } \\
\text { water content ( \% ) }\end{array}$ \\
\hline ‘东方星光’ & 通气对照 CK & $15.29 \pm 0.73$ & $40.70 \pm 0.75$ & $2.60 \pm 0.07$ & $93.61 \pm 0.14$ \\
\hline 'Dongfangxingguang' & 低氧胁迫 Hypoxia stress & $12.26 \pm 0.31$ & $30.09 \pm 0.35$ & $2.03 \pm 0.02$ & $93.25 \pm 0.04$ \\
\hline ‘西域一号’ & 通气对照 CK & $17.77 \pm 0.74$ & $45.10 \pm 0.59$ & $2.75 \pm 0.04$ & $93.91 \pm 0.04$ \\
\hline 'Xiyu 1' & 低氧胁迫 Hypoxia stress & $13.91 \pm 0.44$ & $28.73 \pm 0.57$ & $2.01 \pm 0.03$ & $93.01 \pm 0.05$ \\
\hline
\end{tabular}

2.2 低氧胁迫对幼苗根系活力的影响

由图 1 可以看出,低氧胁迫期间, ‘东方星光’和 ‘西域一号’幼苗的根系活力均呈现先增强后下降的 变化趋势, 且均在低氧胁迫 $4 \mathrm{~d}$ 时达最大值。在低 氧胁迫 $4 \mathrm{~d}$ 时, ‘东方星光’ 根系活力较对照增加 $132.78 \%$, “西域一号”根系活力较对照增加 $104.90 \%$, 低氧胁迫下 ‘东方星光’ 的根系活力稍高 于“西域一号”。

2.3 低氧胁迫对丙酮酸脱羧酶 (PDC) 和乙醇脱氢 酶( $\mathrm{ADH})$ 活性的影响

由图 $2 \mathrm{~A}$ 可以看出, 在低氧胁迫 $2 \mathrm{~d}$ 后, 两品种 根系的 PDC 活性显著升高达最大值, “东方星光” 的
活性为对照的 5.09 倍, “西域一号”为对照的 4.41 倍, 说明低氧胁迫处理显著增强了网纹甜瓜根系 PDC 的活性, 且两品种间无显著差异。随着低氧胁 迫时间的延长, 两品种的 PDC 活性有所下降, 但在 整个处理期间显著高于通气对照。

由图 2B 可以看出, 在低氧胁迫的前 $2 \mathrm{~d}$, 两品种 的 ADH 活性与对照相比无明显变化, 在处理 $4 \mathrm{~d}$ 时, “东方星光”的 $\mathrm{ADH}$ 活性显著上升, 为对照的 34.19 倍, 在处理 $6 \mathrm{~d}$ 时, $\mathrm{ADH}$ 活性达最大值, 以后迅 速下降, $8 \mathrm{~d}$ 时与对照水平相当; 而 “西域一号”处理 $4 \mathrm{~d}$ 后 $\mathrm{ADH}$ 活性开始逐渐升高, 处理 $6 \mathrm{~d}$ 时为对照 的 1.63 倍, $8 \mathrm{~d}$ 时为对照的 2.74 倍。 


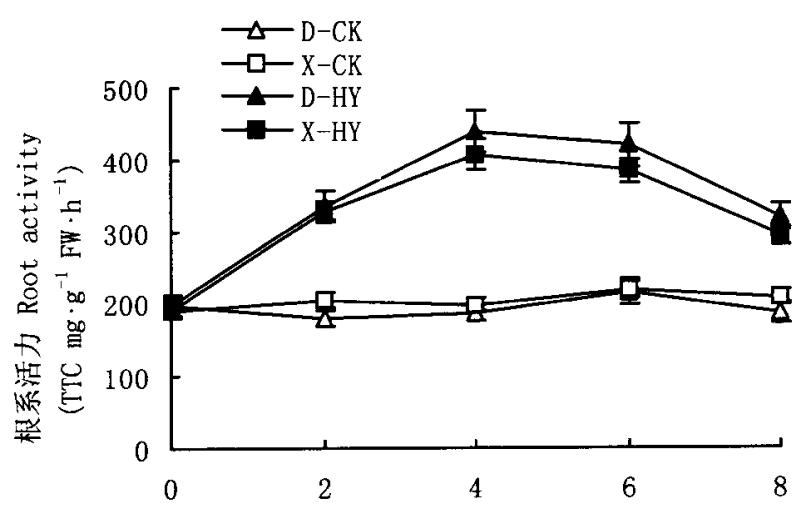

处理天数 Days of treatment

图 1 低氧胁迫对网纹甜瓜幼苗根系活力的影响

Fig. 1 Effect of hypoxia stress on root activity of muskmelon seedling D: ‘东方星光' 'Dongfangxingguang' X: ‘西域 1 号” 'Xiyu 1' HY: 低氧处理 Hypoxia stress CK: 对照 Control
2.4 低氧胁迫对乳酸脱氢酶 $(\mathrm{LDH})$ 和苹果酸脱氢 酶( MDH)活性的影响

由图 3A 可以看出, 随着低氧胁迫时间的延长, “西域一号”的 LDH 活性显著升高, 在低氧胁迫 $2 \mathrm{~d}$ 时为对照的 2.13 倍, 在胁迫 $6 \mathrm{~d}$ 时, $\mathrm{LDH}$ 活性达升 到最高值, 为对照的 4.11 倍, 此后开始下降; 而“东 方星光”在低氧胁迫 $6 \mathrm{~d}$ 时, LDH 活性才显著升高, 为对照的 2.01 倍。

由图 $3 \mathrm{~B}$ 可以看出, 低氧胁迫使两品种根系的 $\mathrm{MDH}$ 活性迅速下降, 在胁迫 $2 \mathrm{~d}$ 时, “东方星光”的 MDH 活性为对照的 $81.99 \%$, “西域一号”为对照的 $58.37 \%$; 在处理 $8 \mathrm{~d}$ “东方星光”的 MDH 活性为对照 的 $68.73 \%$, “西域一号”为对照的 $59.57 \%$, 在低氧 胁迫期间 “东方星光” MDH活性下降的幅度比 “西

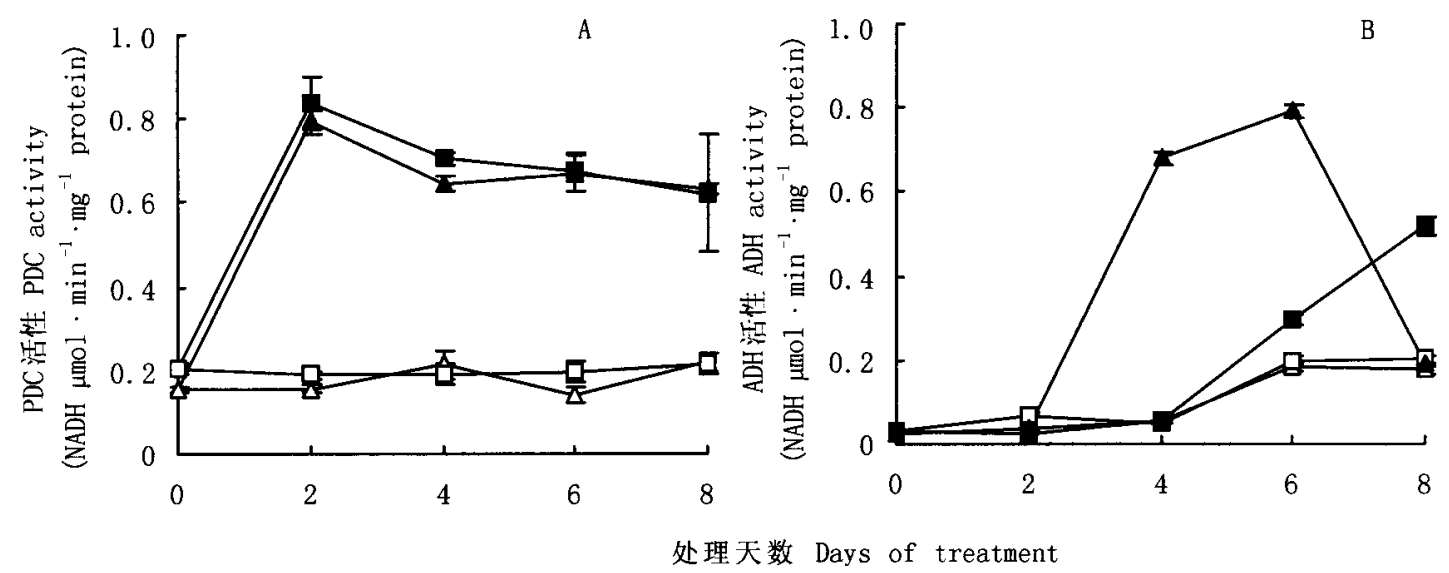

图 2 低氧胁迫对网纹甜瓜根系丙酮酸脱羧酶 $(A)$ 、乙醇脱氢酶 $(B)$ 活性的影响

Fig.2 Effect of hypoxia stress on activities of PDC (A) and ADH (B) in roots of muskmelon seedlings 图例见图 1 Legends see Fig. 1
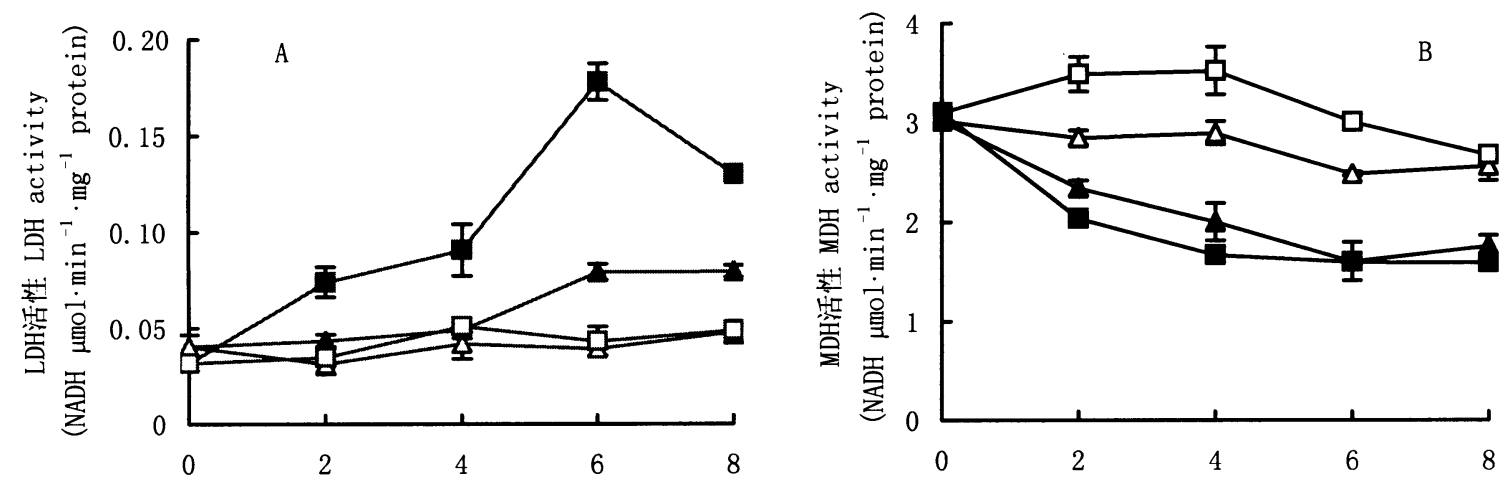

处理天数 Days of treatment 
域一号”小,而 MDH 的活性略高于“西域一号”。

\section{3 讨 论}

根际低氧胁迫下, 植物细胞内氧化磷酸化电子 传递受阻, 造成细胞色素氧化还原系统还原力下降, $\operatorname{ATP}$ 和 $\operatorname{NADP}(H)+$ 生成减少, 抑制植物地上部的光 合作用, 同时阻碍了根系对水分和养分的吸收, 表现 为植物生长缓慢, 生长势减弱, 植株矮小, 根系变褐。 郭世荣 (2000) 研究表明在无氧胁迫下, 黄瓜 ( Cucumis sativus) 和番茄 (Lycopersicon eseulentum) 幼苗全 株鲜重分别为 $4 \mathrm{mg} \cdot \mathrm{L}^{-1} \mathrm{DO}$ 处理的 $57 \%$ 和 $31 \%$; 低 氧胁迫处理的网纹甜瓜幼苗叶片长度和宽度的生长 速率显著低于正常通气对照处理(高洪波和郭世荣, 2005)。本研究结果表明, 低氧胁迫后网纹甜瓜植株 株高、根长显著降低, 地上部、地下部的鲜重和干重 减少, 表明低氧胁迫显著抑制了网纹甜瓜幼苗地上 部和地下部的生长。低氧胁迫下两品种根系活力升 高, 表明根系的脱氢酶活性升高, 这可能是植株适应 低氧胁迫的一种积极性反应。

低氧胁迫下, 植物有氧呼吸受阻, 启动无氧呼 吸, 糖酵解代谢产物丙酮酸通过两条途径产生 ATP 和 $\mathrm{NAD}^{+}$: 丙酮酸在 PDC 作用下生成乙醛, 乙醛在 $\mathrm{ADH}$ 作用下生成乙醇; 丙酩酸在 LDH 作用下直接生 成乳酸。虽然无氧呼吸产生能量效率低, 有机物损 耗大, 但是在低氧胁迫下无氧呼吸可以作为一种临 时补救措施, 维持植株短暂的生长。Guo 等 (1999) 研究指出, 番茄在低氧胁迫下, 根系 ADH、LDH 活性 增加, 糖酵解加速; Mustroph 和 Albrecht (2003) 研究 表明, 低氧胁迫下水稻( Oryza sativa)、小麦、玉米的 根系 PDC、ADH、LDH 活性均显著升高。本试验结果 显示, 低氧胁迫下两品种幼苗根系 PDC、ADH、LDH 活性均有不同程度升高, 说明低氧胁迫下无氧呼吸 代谢途径中酶活性的提高对增强植物的耐低氧能力 起着积极的作用。

无氧呼吸过程中, 乙醛、乙醇、乳酸等代谢产物 积累到一定程度可能对细胞产生毒害, Drew (1997) 提出乙醇在细胞内积累是涝害的主要原因; 但 Perata 和 Alpi( 1993) 研究证明, 乙醇很容易通过细胞膜 脂双层扩散到外部环境中, 而且 ADH 对低氧下种子 的发芽和植物的生存起重要的作用, 因此认为对植 物真正造成伤害的并不是乙醇而是乙醛 (Dennis et $a l ., 2000$ ); Braun 等(1995)研究表明, 乙醛可以和蛋 白形成乙醛一蛋白加合物对细胞造成伤害。Roberts 等(1985)指出, 低氧胁迫下, LDH 活性提高导致乳酸
积累, 造成细胞质酸化, $\mathrm{pH}$ 值降低是涝害、湿害等低 氧条件下植物死亡的主要原因。

Mohanty 和 Ong (2003) 认为 ADH 并不是限制乙 醇发酵的主要因素, 而由于 PDC 的最大催化活性很 小才成为乙醇发酵途径的限制因素 (Morell et al., 1990; Drew, 1997), PDC 活性增加可以提高植物在低 氧胁迫下的耐性, 这在拟南芥 (Arabidopsis thaliana) (Ismond et al.,2003) 和水稻 (Quimio et al., 2000)上 都得到了证实, 原因可能是 PDC 活性的增加使乙醇 发酵生成更多的 ATP 和 $\mathrm{NAD}^{+}$。而本试验表明, 无 论是低氧耐性较强的 “东方星光”, 还是低氧耐性较 弱的“西域一号”, 其 PDC 活性均在处理后两天显著 增加, 达到 $0.8 \mu \mathrm{mol} \cdot \mathrm{min}^{-1} \cdot \mathrm{mg}^{-1}$ protein 左右, 而乙 醇发酵没有超过这一水平, 暗示 PDC 并不是乙醇发 酵的限制因素。

相对于无氧呼吸 $1 \mathrm{~mol}$ 葡萄糖生成 $2 \mathrm{~mol}$ ATP, 而有氧呼吸可以生成 $36 \mathrm{~mol}$ ATP, 因此尽管低氧胁 迫下有氧呼吸较弱, 但是有氧呼吸的进行也可以弥 补低氧胁迫下植物细胞 $\mathrm{ATP} 、 \mathrm{NAD}^{+}$较低对植物造成 的伤害 (潘瑞炽, 2004)。MDH 作为 TCA 循环的一个 重要组成酶, 是调节 TCA 循环速度的控制点之一, 其活性可以反映有氧呼吸的强弱。本试验表明, 正 常条件下 ‘西域一号” MDH 活性比“东方星光”高, 对 氧的依赖性更高, 在低氧下 MDH 活性明显降低, 有 氧呼吸很弱, 不耐低氧; 而“东方星光”在正常条件下 $\mathrm{MDH}$ 活性较低, 对氧的依赖性较低, 在低氧下仍有 较高的 MDH 活性和有氧呼吸, 所以较耐低氧。

不同植物的耐低氧能力不同, 即使同一物种不 同生态型和品种间也存在着明显差异。Guo 和 Tachibana (1997)研究表明当 DO 降到 $1 \mathrm{mg} \cdot \mathrm{L}^{-1}$ 时, 黄瓜植株生长仍可良好生长, 表现出对低氧逆境较 高的耐性, 而番茄植株则在 DO 下降到 $2 \mathrm{mg} \cdot \mathrm{L}^{-1}$ 以 下时就生长不良。高洪波和郭世荣(2004) 研究表 明, 低氧胁迫对不耐低氧的网纹甜瓜伤害较重, 低氧 胁迫后不耐低氧的“西域一号”体内活性氧含量显著 高于耐性较强的 “东方星光”。本试验低氧胁迫后 两品种的 PDC 活性都显著增加, “东方星光” 的 $\mathrm{ADH}$ 活性较高, 可以把乙醛转化成乙醇, 体内不会积累乙 醛, 而 “西域一号”在处理长时间后 $\mathrm{ADH}$ 活性才开始 升高, 可能会造成体内乙醛的临时积累, 这可能是 “西域一号”比“东方星光”不耐低氧的原因之一; 可 以看出, 低氧胁迫下 '东方星光” 以乙醇发酵为无氧 呼吸的主要途径, 而 “西域一号, 以乳酸发酵为无氧 呼吸的主要途径, 后者会造成体内乳酸的积累, 这可 
能是“西域一号”比“东方星光”耐低氧能力弱的原因 之一; 而且在低氧胁迫下“东方星光”根系 MDH 活性 高于 ‘西域一号, 表明耐低氧性较强的品种可以保 持较高的有氧呼吸强度, 这也可能是“东方星光”耐 低氧的重要原因之一。

综上所述, 在低氧胁迫下, 耐低氧性较强的“东 方星光'可以维持较强的有氧呼吸能力, 可以通过乙 醇发酵产生部分能量; 而“西域一号”体内有氧呼吸 能力弱, 加之乳酸发酵产生的乳酸以及体内可能积 累乙醛都会对植株造成伤害, 这是两品种对低氧逆 境耐性差异的主要原因。

\section{参 考 文 献}

Bergmeger H (1983). Methods of Enzymatic Analysis. Verlag Chemse Press, Weinhein, 118 - 125.

Biemelt B, Keetman U, Albrecht G (1998). Re-aeration following hypoxia or anoxia leads to activation of the antioxidative defease system in roots of wheat seedlings. Plant Physiology, 116, 651 -658 .

Bradford MM (1976). A rapid and sentitive method for the quantitation of icrogram quantities of protein utilizing the principle of protein-dye binding. Analytical Biochemistry, 72, $248-254$.

Braun KP, Cody RB, Jones JDR, Peterson CM (1995) . A structural assignment for a stable acetaldehyde-lysine adduct. The Journal of Biological Chemistry, 270, 11263 - 11266.

Dennis ES, Dolferus R, Ellis M, Rahman M, Wu Y, Hoeren FU, Grover A, Ismond KP, Good AG, Peacock WJ (2000). Molecular strategies for improving waterlogging tolerance in plants. Journal of Experimental Botany, 51, 89-97.

Drew MC (1997). Oxygen deficiency and root metabolism: injury and acclimation under hypoxia and anoxia. Annual Review of Plant Physiology and Molecular Biology, 48, $223-250$.

Gao HB (高洪波), Guo SR (郭世荣) (2004). Effects of exogenous $\gamma$-aminobutyric acid on antioxidant enzyme ativity and reactive oxygen content in muskmelon seedlings under nutrient solution hypoxia stress. Journal of Plant Physiology and Molecular Biology (植物生理与分子生物学学报), 30, 651-659. (in Chinese with English abstract)

Gao HB (高洪波), Guo SR (郭世荣) (2005). Effect of hypoxia stress on polyamines contents of muskmelon seedlings with hydroponics. Acta Horticulturae Sinica（园艺学报), 32, 121 - 123. (in Chinese with English abstract)

Guo SR (郭世荣) (2000). Effect of dissolved oxygen concentrations in nutrient solution on the respiratory intensity of cucumber and tomato roots. Acta Horticulturae Sinica (园艺学报), 27, 141 - 142. (in Chinese with English abstract)

Guo SR (郭世荣) (2003). Soilloss Culture (无土栽培学). Chi- na Agriculture Press, Beijing, 125 - 127. (in Chinese)

Guo SR, Nada K, Katoh H, Tachibana S (1999). Differences between tomato (Lycopersicon esculentum Mill.) and cucumber (Cucumis sativus L.) in ethanol, lactate and malate metabolisms and cell sap $\mathrm{pH}$ of roots under hypoxia. Journal of the Japanese Society for Horticultural Science, 68, 152 - 159.

Guo SR, Tachibana SJ (1997). Effect of dissolved $\mathrm{O}_{2}$ levels in a nutrient of tomato and mineral nutrition of tomato and cucumber seedlings. Journal of the Japanese Society for Horticultural Science, 66, $331-337$.

Ismond KP, Dolferus R, Pauw MD, Dennis ES, Good AG (2003) . Enhanced low oxygen survival in Arabidopsis through increased metabolic flux in the fermentative pathway. Plant Physiology, $132,1292-1302$.

Johnson JR, Cobb BG, Drew MC (1994). Hypoxic induction of anoxia tolerance in roots of adh1 null Zea mays L. Plnat Physiology, 105, $61-67$.

Li HS (李合生) (2000). Principles and Techniques of Plant Physiological Biochemical Experiment (植物生理生化实验原理和 技术). Higher Education Press, Beijing, 119-120. (in Chinese)

Mohanty B, Ong BL (2003) . Contrasting effects of submergence in light and dark on pyruvate decarboxylase activity in roots of rice lines differing in submergence tolerance. Annals of Botany, 91, $291-300$.

Morrell S, Greenway H, Davies DD (1990). Regulation of pyruvate decarboxylase in vitro and in vivo. Journal of Experimental Botany, 41, 131 - 139 .

Mustroph A, Albrecht G (2003). Tolerance of crop plants to oxygen deficiency stress: fermentative activity and photosynthetic capacity of entire seedlings under hypoxia and anoxia. Physiologia Plantarum, 117, $508-520$.

Pan RC (潘瑞炽) (2004). Plant Physiology (植物生理学) 5th edn. Higher Education Press, Beijing ,102-115. (in Chinese)

Perata P, Alpi A (1993). Plant responses to anaerobiosis. Plant Science, 93, 1-17.

Quimio CA, Torrizo LB, Setter TL, Ellis M, Grover A, Abrigo EM, Oliva NP, Ella ES, Carpena AL, Ito O (2000). Enhancement of submergence tolerance in transgenic rice overproducing pyruvate decarboxylase. Journal of Plant Physiology, 156, 516 -521 .

Roberts JKM, Andrade FH, Anderson IC (1985) . Further evidence that cytoplasmic acidosis is a determinant of flooding intolerance in plants. Plant Physiology, 77, $492-494$.

Waters I, Morell S, Greenway H, Colmer TD (1991). Effect of anoxia on wheat seedlings. II . Influence of $\mathrm{O}_{2}$ supply prior to anoxia on tolerance to anoxia, alcoholic fermentation, and sugar levels. Journal of Experimental Botany, 42, 1437 - 1447.

Xue YL (薛应龙) (1985)。 Plant Physiology Experimental Enchiridion (植物生理学实验手册). Shanghai Scientific and Technical Publisher, Shanghai, 179 - 181. (in Chinese) 